\title{
Thickness dependence of dielectric properties in bismuth layer-structured dielectrics
}

\author{
Kenji Takahashi, Muneyasu Suzuki, and Takashi Kojima \\ Department of Innovative and Engineered Materials, Tokyo Institute of Technology, 4259 Nagatsuta, \\ Midori-ku, Yokohama 226-8502, Japan \\ Takayuki Watanabe \\ Department of Innovative and Engineered Materials, Tokyo Institute of Technology, 4259 Nagatsuta, \\ Midori-ku, Yokohama 226-8502, Japan and Institute of Solid State Research, Research Center \\ and Center of Nanoelectronic Systems for Information Technology, Research Center Juelich, \\ D-52425 Juelich, Germany \\ Yukio Sakashita \\ TDK Corporation, 570-2 Matsugashita, Minamihatori, Narita-shi, Chiba 286-8588, Japan
}

Kazumi Kato

National Institute of Advanced Industrial Science and Technology, 2266-98 Anagahora, Shimoshidami, Moriyama-ku, Nagoya 463-8560, Japan

Osami Sakata and Kazushi Sumitani

Materials Science Division, Japan Synchrotron Radiation Research Institute (JASRI)/SPring-8, Kouto, Mikazuki, Sayo, Hyogo 679-5198, Japan

Hiroshi Funakubo ${ }^{\text {a) }}$

Department of Innovative and Engineered Materials, Tokyo Institute of Technology, 4259 Nagatsuta, Midori-ku, Yokohama 226-8502, Japan and PRESTO, JST, 4-1-8 Honmachi, Kawaguchi, Saitama 332-0012, Japan

(Received 6 February 2006; accepted 8 July 2006; published online 21 August 2006)

$c$-axis-oriented epitaxial $\mathrm{SrBi}_{4} \mathrm{Ti}_{4} \mathrm{O}_{15}$ and $\mathrm{CaBi}_{4} \mathrm{Ti}_{4} \mathrm{O}_{15}$ films having natural superlattice structure were grown on $(001){ }_{c} \mathrm{SrRuO}_{3} \|(001) \mathrm{SrTiO}_{3}$ substrates by metal organic chemical vapor deposition. $\mathrm{SrBi}_{4} \mathrm{Ti}_{4} \mathrm{O}_{15}$ films suffer no degradation with a dielectric constant of 200 down to a film thickness of $15 \mathrm{~nm}$, which corresponds to four unit cells. Temperature coefficients of capacitance were low enough despite their high dielectric constant. They exhibited stable capacitance and superior insulating properties against applied electric field, irrespective of film thickness. These results open the door to designable size-effect-free materials with high dielectric constant having bias- and temperature-independent characteristics together with superior electrical insulation for high-density capacitor applications. (C) 2006 American Institute of Physics. [DOI: 10.1063/1.2336626]

Simple perovskite-structured oxides, especially $(\mathrm{Ba}, \mathrm{Sr}) \mathrm{TiO}_{3}$, have been investigated as promising candidates for the integrated capacitor application. ${ }^{1-4}$ However, the significant drop in the dielectric constant with decreased film thickness, widely known as "size effect," has been reported to become serious. ${ }^{1-4}$ The size effect in thin film dielectrics has made it difficult to design their performance characteristics. There has therefore been a strong motivation to discover size-effect-free highly insulating dielectric materials with a high dielectric constant. In this letter, we propose thin films with $c$-axis-oriented bismuth layer-structured dielectrics (BLDs) as candidates for size-effect-free materials.

BLDs have a natural superlattice structure along the $c$ axis consisting of two kinds of two-dimensional nanolayers, i.e., a bismuth oxide $\left(\mathrm{Bi}_{2} \mathrm{O}_{2}\right)^{2+}$ sheet and a pseudoperovskite block generally described as $\left(A_{m-1} B_{m} \mathrm{O}_{3 m+1}\right)^{2-}$, where $m$ is the number of $\mathrm{BO}_{6}$ octahedra in a pseudoperovskite block. ${ }^{5}$ Interestingly, BLDs with even $m$ exhibit no ferroelectricity along the $c$ axis. ${ }^{5,6}$

In our previous study, we found that $c$-axis-oriented epitaxial $\mathrm{SrBi}_{2} \mathrm{Ta}_{2} \mathrm{O}_{9}(\mathrm{SBT}, m=2)$ films exhibited no degradation in dielectric constant down to $20 \mathrm{~nm}$ in thickness, cor-

${ }^{a)}$ Electronic mail: funakubo@iem.titech.ac.jp responding to eight unit cells. ${ }^{7}$ However, their dielectric constant of 55 was obviously not enough to replace the conventional perovskite-structured oxides. We therefore expanded our concept to a higher $m$ of 4 , i.e., $\mathrm{SrBi}_{4} \mathrm{Ti}_{4} \mathrm{O}_{15}$ (SBTi) and $\mathrm{CaBi}_{4} \mathrm{Ti}_{4} \mathrm{O}_{15}(\mathrm{CBTi})$, to obtain higher dielectric constant and to deepen the understanding of the basic characteristics of $c$-axis-oriented BLD films. As a result, we found the design concept of their properties and revealed that thin films of $c$-axis-oriented BLDs are promising candidates for high-density capacitor application.

$c$-axis-oriented epitaxial $\mathrm{SBTi}$ and $\mathrm{CBTi}$ films were grown at a substrate temperature of $700{ }^{\circ} \mathrm{C}$ by metal organic chemical vapor deposition. (001) $c_{c}$-oriented epitaxial $\mathrm{SrRuO}_{3}$ (SRO) films having an atomically flat surface grown on (001)STO single crystals were used as substrates. ${ }^{8}$

The constituent phase and the orientation were identified by conventional $\mathrm{x}$-ray diffraction (XRD). The crystal structure was analyzed in detail by XRD using synchrotron radiation (SPring-8, BL13XU). ${ }^{9}$ The dielectric and insulation properties of the films were measured after formation of the top electrodes of $100 \mu \mathrm{m} \varphi \mathrm{Pt}$ on the film surface followed by postannealing in an electrical furnace at $400{ }^{\circ} \mathrm{C}$ for $30 \mathrm{~min}$ under $1 \mathrm{~atm}$ flowing oxygen ambience. 


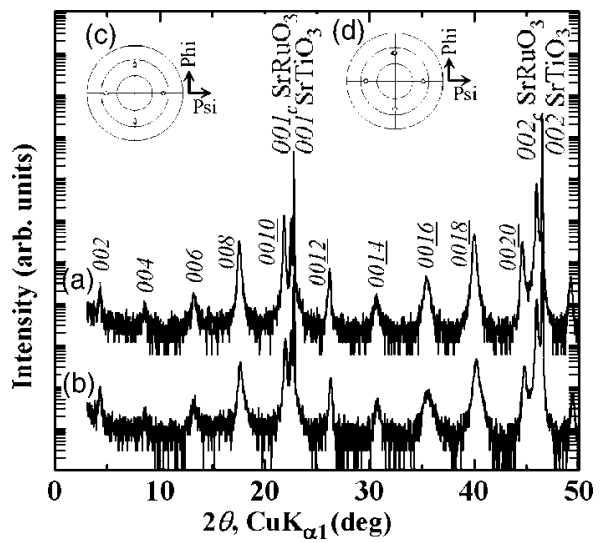

FIG. 1. XRD $\theta-2 \theta$ patterns of 98 and $85 \mathrm{~nm}$ thick (a) $\mathrm{SrBi}_{4} \mathrm{Ti}_{4} \mathrm{O}_{15}$ and (b) $\mathrm{CaBi}_{4} \mathrm{Ti}_{4} \mathrm{O}_{15}$ films grown on $(001)_{c} \mathrm{SrRuO}_{3} \|(001) \mathrm{SrTiO}_{3}$ substrates. [(c) and (d)] X-ray pole figures of the same $\mathrm{SrBi}_{4} \mathrm{Ti}_{4} \mathrm{O}_{15}$ and $\mathrm{CaBi}_{4} \mathrm{Ti}_{4} \mathrm{O}_{15}$ films, where the fixed $2 \theta$ angle corresponds to the 119 reflection for each phase.

Figure 1 shows typical XRD patterns for 98 and $85 \mathrm{~nm}$ thick (a) SBTi and (b) CBTi films grown on $(001)_{c} \mathrm{SRO} \|(001) \mathrm{STO}$ substrate. Only the $002 l$ ( $l=$ integer number) peaks of each BLD phase were observed together with those from the substrate. The epitaxial growth of these films was confirmed by x-ray pole figure measurements.

Figure 2(a) plots the dependence of surface normal and in-plane lattice parameters on film thickness, and the unit cell volume of epitaxial SBTi films, where each value indicates the change normalized by the reported values for

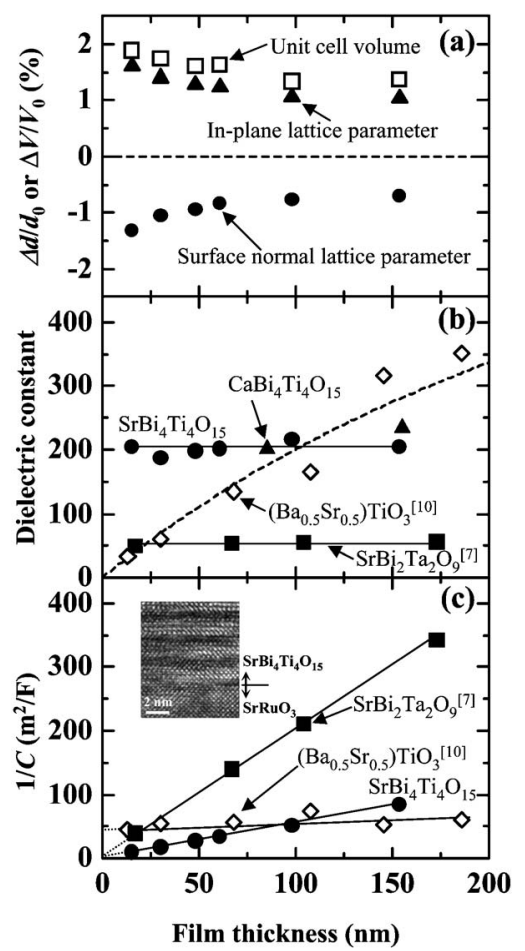

FIG. 2. (a) Thickness dependencies of surface normal $(\mathbf{O})$ and in-plane $(\mathbf{\Delta})$ lattice parameters, and unit cell volume $(\square)$ of $\mathrm{SrBi}_{4} \mathrm{Ti}_{4} \mathrm{O}_{15}$ films. Each value was normalized by the strain-free powder $\operatorname{data}\left(d_{0}\right.$ and $\left.V_{0}\right)$. (b) Thickness dependence of the dielectric constant of $\mathrm{SrBi}_{4} \mathrm{Ti}_{4} \mathrm{O}_{15}(-)$ and $\mathrm{CaBi}_{4} \mathrm{Ti}_{4} \mathrm{O}_{15}(\mathbf{\Delta})$ films. (c) Reciprocal capacitance as a function of thickness of $\mathrm{SrBi}_{4} \mathrm{Ti}_{4} \mathrm{O}_{15}$ films (-). Data for $\mathrm{SrBi}_{2} \mathrm{Ta}_{2} \mathrm{O}_{9}$ (Ref. 7) (汭) and $\left(\mathrm{Ba}_{0.5} \mathrm{Sr}_{0.5}\right) \mathrm{TiO}_{3}(\diamond)($ Ref. 10) films are also shown in (b) and (c) for comparison. Inset in (c) is a cross-sectional HRTEM image near the interface between the $15 \mathrm{~nm}$ thick $\mathrm{SrBi}_{4} \mathrm{Ti}_{4} \mathrm{O}_{15}$ film and $\mathrm{SrRuO}_{3}$ bottom electrode layer.

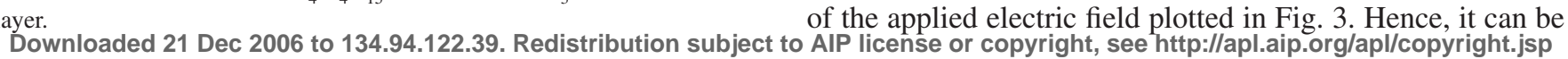

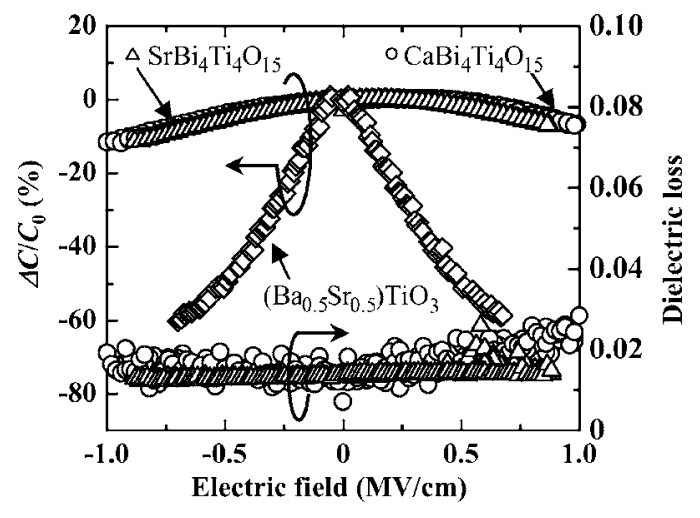

FIG. 3. Capacitance change and dielectric loss as a function of applied electric field for the same films shown in Fig. 1 together with those reported for $\left(\mathrm{Ba}_{0.5} \mathrm{Sr}_{0.5}\right) \mathrm{TiO}_{3}$ film (Ref. 2). $C_{0}$ corresponds to the zero capacitance.

strain-free powder. The in-plane lattice parameter is larger than that of powder for all films and gradually increases as film thickness decreases, indicating an increased in-plane residual strain. For $17 \mathrm{~nm}$ films, it is almost identical to that of strained $\mathrm{SrRuO}_{3}$ bottom electrodes grown on $(001) \mathrm{SrTiO}_{3}$ substrates. This indicates that the film is fully strained. The surface normal lattice parameter, on the other hand, decreased with the increase in the in-plane parameter to maintain the unit cell volume. However, the unit cell volume increased with decreased film thickness as can be seen in Fig. 2(a). The difference of lattice parameters and unit cell between the film and the powder shown in Fig. 2(a) could be solely attributed to the misfit strain during film deposition. Figure 2(b) plots the dielectric constant of $c$-axis-oriented epitaxial SBTi and CBTi films as a function of the thickness, as well as those for epitaxial SBT films ${ }^{7}$ and reported data on epitaxial $\left(\mathrm{Ba}_{0.5} \mathrm{Sr}_{0.5}\right) \mathrm{TiO}_{3}$ films ${ }^{10}$ for comparison. The dielectric constant of SBTi and CBTi films was almost 200 irrespective of the kind of cations in the pseudoperovskite blocks. These values were around four times larger than those of SBT films with $m$ of 2, suggesting that the dielectric constant of BLDs was mainly determined by $m$. Moreover, SBTi films did not exhibit any degradation in dielectric constant by decreasing film thickness down to $15 \mathrm{~nm}$, which corresponds to four unit cells $(c=4.040 \mathrm{~nm})$, indicating that dielectric properties are independent of film thickness, i.e., no size effect. This clearly indicates that in-plane tensile stress applied to $c$-axis-oriented SBTi films did not greatly affect their dielectric characteristics.

Furthermore, film thickness dependency of the reciprocal of capacitance for SBTi films shows the linear relationship across the zero point as shown in Fig. 2(c), suggesting nonexistence of the dead layer, which is pointed out to lead to the size effect. ${ }^{11}$ Here, no obvious interlayer could be observed in the cross-sectional high-resolution transmission electron microscopy (HRTEM) image of the interface between the $15 \mathrm{~nm}$ thick SBTi film and the SRO bottom electrode layer as shown in the inset of Fig. 2(c).

Figure 3 plots capacitance change and dielectric loss as a function of the applied electric field for the same films as in Fig. 1 together with the reported ones for $\left(\mathrm{Ba}_{0.5} \mathrm{Sr}_{0.5}\right) \mathrm{TiO}_{3}$ film. ${ }^{2}$ The changes in capacitance between 0 and $900 \mathrm{kV} / \mathrm{cm}$ for SBTi and CBTi films were $7.0 \%$ and $6.5 \%$, respectively, while that for $\left(\mathrm{Ba}_{0.5} \mathrm{Sr}_{0.5}\right) \mathrm{TiO}_{3}$ film was $59 \%$. In addition, the dielectric loss was less than $3 \%$, which was also independent 


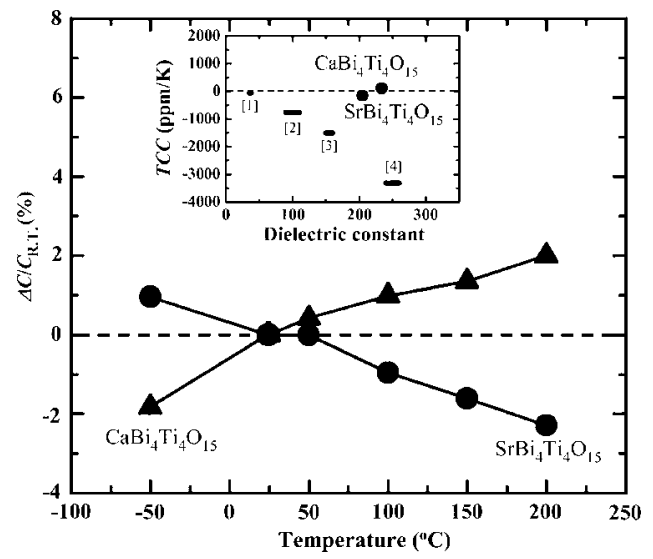

FIG. 4. Capacitance change $\left(\Delta C / C_{\mathrm{RT}}\right)$ as a function of temperature for the same films shown in Fig. 1. $C_{\mathrm{RT}}$ stand for capacitance at room temperature $\left(25^{\circ} \mathrm{C}\right)$. Inset shows the temperature coefficient of capacitance (TCC) as a function of dielectric constant, where data for typical dielectric materials (Ref. 13) ([1] ZnO- $\mathrm{TiO}_{2}$, [2] $\mathrm{TiO}_{2},[3] \mathrm{CaTiO}_{3}$, and [4] $\mathrm{SrTiO}_{3}$ ) are also shown for comparison.

concluded from Fig. 3, combined with our previous results of SBT films, ${ }^{7}$ that this stable capacitance against applied electric field is a common feature of $c$-axis-oriented BLD films irrespective of $m$.

Figure 4 plots the dependence of capacitance change with temperature normalized at room temperature for the same SBTi and CBTi films shown in Figs. 1(a) and 1(b). No obvious dependence on film thickness was observed for the thickness range in Fig. 2. The temperature coefficients of the capacitance (TCCs) from -50 to $150{ }^{\circ} \mathrm{C}$ were -160 and $+110 \mathrm{ppm} / \mathrm{K}$, respectively. These TCCs are sufficiently low compared with those reported for $\left(\mathrm{Ba}_{0.7} \mathrm{Sr}_{0.3}\right) \mathrm{TiO}_{3}$ films, i.e., $-833 \mathrm{ppm} / \mathrm{K}{ }^{12}$ Taking into account the fact that the TCC value is generally negatively proportional to the dielectric constant as shown in the inset of Fig. $4,{ }^{13}$ these results are quite unique, indicating low TCC values are consistent with a high dielectric constant. Furthermore, zero TCC can be expected to be achieved by making a solid solution of SBTi and CBTi.

Figure 5 plots the leakage current density at an applied electric field of $500 \mathrm{kV} / \mathrm{cm}$ for epitaxial SBTi and CBTi films, They were almost constant below $10^{-6} \mathrm{~A} / \mathrm{cm}^{2}$ irrespective of the film thickness and the applied electric field. Note that superior electric insulation was achieved in film as thin as $15 \mathrm{~nm}$, as shown in the inset of Fig. 5, even though it was grown on a conductive oxide electrode, which has been reported to degrade the insulation properties of the dielectric films. ${ }^{14}$ Given the fact that Kim et al. pointed out that the $\left(\mathrm{Bi}_{2} \mathrm{O}_{2}\right)^{2+}$ layers act as an insulating barrier, ${ }^{15}$ the natural superlattice structure consisting of a $\left(\mathrm{Bi}_{2} \mathrm{O}_{2}\right)^{2+}$ layer and a pseudoperovskite block along the $c$ axis, i.e., perpendicular to the applied electric field, can be considered to be the key to producing highly effective electric insulation in $c$-axis-oriented BLD films.

The results of the present study suggest that filmthickness-independent insulating and dielectric characteristics, and electric-field-independent stable capacitance are common features of BLDs with even $m$. Tensile strain applied to the present BLDs must be carefully discussed for the next step. However, the results on the present study clearly reveal that films with BLDs with even $m$ are alternative candidates to conventional high dielectric constant materials,

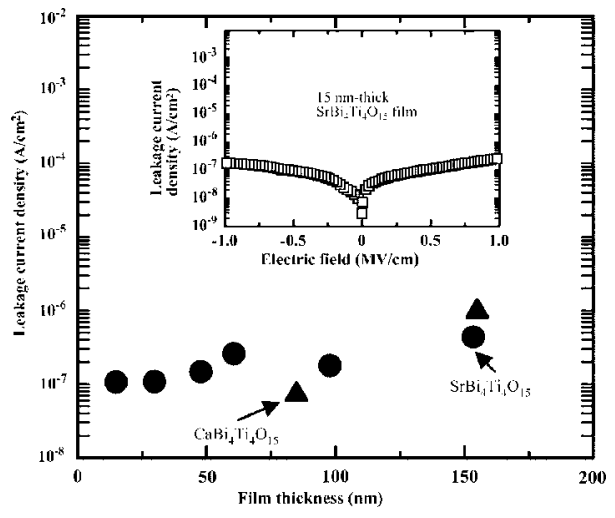

FIG. 5. Leakage current density measured at $500 \mathrm{kV} / \mathrm{cm}$ as a function of film thickness of $c$-axis-oriented epitaxial $\mathrm{SrBi}_{4} \mathrm{Ti}_{4} \mathrm{O}_{15}(\mathcal{O})$ and $\mathrm{CaBi}_{4} \mathrm{Ti}_{4} \mathrm{O}_{15}$ (A) films. Inset shows leakage current dependence on applied electric field of a $15 \mathrm{~nm}$ thick $\mathrm{SrBi}_{4} \mathrm{Ti}_{4} \mathrm{O}_{15}$ film.

such as $(\mathrm{Ba}, \mathrm{Sr}) \mathrm{TiO}_{3}$, for thin film capacitors because they are free of the size effect and have a high dielectric constant with good stability against electric field and temperature. These results open the door to designable size-effect-free materials with high dielectric constant having bias- and temperature-independent characteristics together with superior electrical insulation for high-density capacitor applications.

In summary, $c$-axis-oriented SBTi and CBTi films were epitaxially grown on $(001){ }_{c}$ SRO $\|(001)$ STO substrates by MOCVD. The dielectric constant of these films maintained a constant value of 200 irrespective of the film thickness down to $15 \mathrm{~nm}$, indicating dielectric characteristics that were free of the size effect. The capacitances of these films were also independent of applied electric field and temperature. The leakage current densities were superior to the constant on the order of $10^{-7} \mathrm{~A} / \mathrm{cm}^{2}$ at $500 \mathrm{kV} / \mathrm{cm}$ regardless of the film thickness. These characteristics are, therefore, very attractive for high-density capacitor applications.

${ }^{1}$ C. S. Hwang, S. O. Park, C. S. Kang, H.-J. Cho, H.-K. Kang, S. T. Ahn, and M. Y. Lee, Jpn. J. Appl. Phys., Part 1 34, 5178 (1995).

${ }^{2}$ P. Padmini, T. R. Taylor, M. J. Lefevre, A. S. Nagra, R. A. York, and J. S. Speck, Appl. Phys. Lett. 75, 3186 (1999).

${ }^{3}$ S. Ezhilvalavan and T.-Y. Tseng, Mater. Chem. Phys. 65, 227 (2000).

${ }^{4}$ L. J. Sinnamon, R. M. Bowman, and J. M. Gregg, Appl. Phys. Lett. 78, 1724 (2001).

${ }^{5}$ B. Aurivillius, Ark. Kemi 1, 463 (1949); Ark. Kemi 1, 499 (1949).

${ }^{6}$ R. E. Newnham, R. W. Wolfe, and J. F. Dorrian, Mater. Res. Bull. 6, 1029 (1971).

${ }^{7}$ T. Kojima, Y. Sakashita, T. Watanabe, K. Kato, and H. Fuankubo, Mater. Res. Soc. Symp. Proc. 748, U15.2.1 (2003).

${ }^{8}$ A. Sumi, K. Takahashi, S. Yokoyama, H. Morioka, H. Funakubo, and M. Yoshimoto, Appl. Phys. Lett. 87, 052112 (2005).

${ }^{9}$ O. Sakata, Y. Furukawa, S. Goto, T. Mochizuki, T. Uruga, K. Takeshita, H. Ohashi, T. Ohata, T. Matsushita, S. Takahashi, H. Tajiri, T. Ishikawa, M. Nakamura, M. Ito, K. Sumitani, T. Takahashi, T. Shimura, A. Saito, and M. Takahasi, Surf. Rev. Lett. 10, 543 (2003). The synchrotron X-ray measurements were performed under JASRI J05A13XU-0510N.

${ }^{10}$ L. J. Sinnamon, M. M. Saad, R. M. Bowman, and J. M. Gregg, Appl. Phys. Lett. 81, 703 (2002).

${ }^{11}$ S. K. Streiffer, C. Basceri, C. B. Parker, S. E. Lash, and A. I. Kingon, J. Appl. Phys. 86, 4565 (1999).

${ }^{12}$ C. B. Parker, J.-P. Maria, and A. I. Kingon, Appl. Phys. Lett. 81, 340 (2002).

${ }^{13}$ Handbook of Ceramics, 2nd ed., edited by The Ceramic Society of Japan (Gihodo, Tokyo, 2002), p. 1006 (in Japanese).

${ }^{14}$ Y. C. Choi and B. S. Lee, Mater. Chem. Phys. 61, 124 (1999).

${ }^{15}$ S.-K. Kim, M. Miyayama, and H. Yanagida, Mater. Res. Bull. 31, 121 (1996). 